\title{
A unique cluster of roo insertions in the promoter region of a stress response gene in Drosophila melanogaster
}

\author{
Miriam Merenciano ${ }^{1}$, Camillo lacometti ${ }^{1,2}$ and Josefa González ${ }^{1 *}$ (D)
}

\begin{abstract}
Transposable elements (TEs) are not randomly distributed in the genome. A genome-wide analysis of the $D$. melanogaster genome found that differences in TE density across $50 \mathrm{~kb}$ genomic regions was due both to transposition and duplication. At smaller genomic scales, promoter regions of hsp genes and the promoter region of CG18446 have been shown to accumulate TE insertions. In this work, we have further analyzed the promoter region of CG18446. We screened 218 strains collected in 15 natural populations, and we found that the CG18446 promoter region contains 20 independent roo insertions. Based on phylogenetic analysis, we suggest that the presence of multiple roo insertions in this region is likely to be the result of several bursts of transposition. Moreover, we found that the roo insertional cluster in the CG18446 promoter region is unique: no other promoter region in the genome contains a similar number of roo insertions. We found that, similar to hsp gene promoters, chromatin accessibility could be one of the factors explaining the recurrent insertions of roo elements in CG18446 promoter region.
\end{abstract}

Keywords: Transposable element, Fecundity, Viability, Target site duplication, Recurrent insertion, Natural population

\section{Background}

Recurrent insertion of transposable elements in specific genomic regions has been described in the Drosophila melanogaster reference genome. The analysis of $50 \mathrm{~kb}$ genomic windows identified 23 regions with a high density of TE insertions, most of them located in pericentromeric regions or on chromosome 4 [1]. Transposition and duplication were identified as the two mechanisms generating these high-density $\mathrm{TE}$ regions. In recent years, computational pipelines have been developed to analyze the TE content in multiple strains [2-4]. Thus, besides TEs annotated in the reference genome, non-reference TE insertions can now also be analyzed. Based on these population analyses, some genes have also been reported to accumulate many TE insertions, such as the $106.5 \mathrm{~kb}$ klarsicht, and the $24 \mathrm{~kb}$ derailed-2 that were analyzed in 146 strains of the Drosophila Synthetic Population Resource [5, 6]. At a much finer scale, several insertions in the proximal promoter regions of

\footnotetext{
* Correspondence: josefa.gonzalez@ibe.upf-csic.es

${ }^{1}$ Institute of Evolutionary Biology (CSIC-Universitat Pompeu Fabra), Passeig

Maritim de la Barceloneta 37,49, 08003 Barcelona, Spain

Full list of author information is available at the end of the article
}

$h s p$ genes have been reported $[7,8]$. While the vast majority of these insertions were P-elements, insertions from the Gypsy and the Jockey family were also identified. P-elements have a preference to insert in $5^{\prime}$ gene flanking regions [9]. The accumulation of TEs in the promoter of $h s p$ genes was explained by the chromatin conformation of this particular region, and by selection favoring the retention of TEs because of their effect on gene expression [8]. More recently, nine roo insertions were also described in the promoter region of another stress response gene, CG18446 that encodes a nucleic acid binding protein [10]. CG18446 is a cold resistance candidate gene [11] and an ethanol-regulated gene [12] highly expressed in ovaries and in 6-10 h-old embryos [13]. Only one of the nine identified insertions was found to consistently affect the expression of CG18446, and it was associated with increased viability in nonstress and cold-stress conditions [10]. However, only 39 strains from two natural populations were screened, and thus it is still an open question whether more roo insertions are present in the CG18446 promoter region. Indeed, roo are the most abundant elements in the $D$. melanogaster genome $[14,15]$. Thus, it is possible that

(c) The Author(s). 2019 Open Access This article is distributed under the terms of the Creative Commons Attribution 4.0 International License (http://creativecommons.org/licenses/by/4.0/), which permits unrestricted use, distribution, and 
besides the cluster identified by Merenciano et al. (2016) [10] other similar clusters of roo insertions in gene promoter regions are present in the genome. Interestingly, while the majority of strains analyzed so far contain a roo insertion (26 out of 39), none of them contains more than one insertion [10].

In this work, we looked for TE insertions in the CG18446 promoter region in 218 strains from 15 natural populations in Europe, North America, and Africa. In addition, based on the analysis of the reference genome, and on the analysis of 177 DGRP strains, we identified 53 promoter regions that could potentially contain multiple roo insertions. Finally, we performed fecundity and viability experiments to investigate why we did not find any fly containing two roo insertions in the CG18446 promoter region.

\section{Results}

Twenty roo solo LTR insertions are present in the CG18446 promoter region in natural populations

To check whether there were more roo insertions in the CG18446 promoter region, we performed a PCR screening in 218 strains from 15 natural populations: 13 European, one North American [16], and one African population collected in the ancestral range of the species (Zambia) (Additional file 1) [17]. 143 strains gave a band consistent with the presence of an insertion, in homozygous or heterozygous state, and 75 strains gave a band consistent with the absence of an insertion (Table 1 and Additional file 2A). We sequenced all the obtained PCR bands and we found that besides the nine insertions discovered in Merenciano et al. (2016) [10], there are 11 other $428 \mathrm{bp}$ roo solo-LTR insertions in the promoter region of CG18446 (Fig. 1). All the strains with an insertion contained a single roo insertion. Across strains, three of the insertion sites contained roo elements inserted in opposite orientations, roo-90, FBti0019985 and $\mathrm{roo}_{+}$, suggesting recurrent insertion in the same exact genomic position (Fig. 1). Recurrent insertion in the same exact genomic position has also been described for P-elements in D. melanogaster [18, 19]. Note that based on the results of T-lex2 [20], a computational pipeline that estimates presence/absence of insertions based on next generation sequencing data, we previously reported that the first nine roo insertions described in the CG18446 promoter region were present in Zambia [10]. However, PCR analyses of 23 of the 42 strains analyzed with T-lex 2 containing four of these nine insertions indicated that these four insertions are not actually present in any of the strains (Additional file 2B). These four unvalidated insertions were polymorphic according to T-lex2. Thus, it could be that these insertions

Table 1 PCR results and roo insertions identified in the 218 strains analyzed in this work, and in brackets insertions identified in the 39 strains analyzed in Merenciano et al. (2016) (10)

\begin{tabular}{|c|c|c|c|c|}
\hline Population & Strains analyzed & $\begin{array}{l}\text { Strains homozygous for the } \\
\text { presence of a roo insertion }\end{array}$ & $\begin{array}{l}\text { Strains heterozygous for the } \\
\text { presence of a roo insertion }\end{array}$ & roo insertions identified \\
\hline Akka, Fl & 13 & 3 & 4 & $\mathrm{roO}-90, \mathrm{rOO}_{-64}, \mathrm{roO}-291$ \\
\hline Stockholm, SE & 23 & 9 & 6 & $\begin{array}{l}\mathrm{rOO}_{-44,} \mathrm{rOO}_{-68}, \mathrm{rOO}_{-90}, \mathrm{rOO}_{-393,} \mathrm{rOO}_{-64}, \mathrm{rOO}_{-42} \\
\text { FBtio019985 } \\
\left(3^{\prime}-5^{\prime}\right)\end{array}$ \\
\hline Lund, SE & 6 & 3 & 1 & $r o o_{-68}, \mathrm{roO}_{-64}$ \\
\hline Karensminde, DK & 12 & 5 & 2 & FBtio019985, roo $19, \mathrm{rOO}_{-68}, \mathrm{roO}_{-64}, \mathrm{rOO}_{-90\left(3^{\prime}-5^{\prime}\right)}$ \\
\hline Munich, DE & 14 & 6 & 5 & $\mathrm{rOO}_{+} 175, \mathrm{rOO}_{-68}, \mathrm{rOO}_{-90}, \mathrm{rOO}_{-378}$ \\
\hline $\begin{array}{l}\text { Market Harborough, } \\
\text { UK }\end{array}$ & 20 & 5 & 7 & $\begin{array}{l}\text { FBtiOO } 9985, \mathrm{rOO}_{+} 37, \mathrm{rOO}_{-68}, \mathrm{rOO}_{-90}, \mathrm{rOO}_{-2} 291, \mathrm{rOO}_{-} \\
42, \mathrm{rOO}_{-90\left(3^{\prime}-5^{\prime}\right)}\end{array}$ \\
\hline Gotheron, FR & 13 & 3 & 2 & $\mathrm{rOO}_{-68}, \mathrm{rOO}_{-90}, \mathrm{rOO}_{-378}, \mathrm{rOO}_{-64}$ \\
\hline Bari, IT & (12) & (3) & (4) & FBtiool9985, roo+ $175, \mathrm{rOO}_{-19}, \mathrm{rOO}_{-28}, \mathrm{roO}_{-68,} \mathrm{roO}_{-90}$ \\
\hline Gimenells, ES & 14 & 3 & 9 & FBtioo19985, $\mathrm{roO}_{+} 175, \mathrm{rOO}_{-44}, \mathrm{rOO}_{-90}$ \\
\hline Tomelloso, ES & 15 & 3 & 10 & $r o o_{-44}, r o o_{-90}, r o o_{-291}$ \\
\hline Cortes de Baza, ES & 13 & 0 & 9 & $r O O_{-44}, r O O_{-90}, r O O_{-90}\left(3^{\prime}-5^{\prime}\right)$ \\
\hline Guadix, ES & 14 & 0 & 11 & 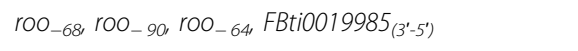 \\
\hline $\begin{array}{l}\text { San Cristóbal de la } \\
\text { Laguna, ES }\end{array}$ & 12 & 6 & 2 & FBtiO019985, roo-90, roo- 291 \\
\hline Raleigh, US & $22(27)$ & $17(19)$ & 0 & 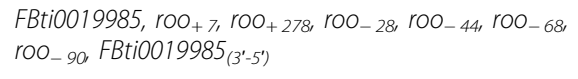 \\
\hline Siavonga, Zl & 27 & 2 & 10 & $\mathrm{rOO}-90, \mathrm{rOO}_{+} 7\left(3^{\prime}-5^{\prime}\right), \mathrm{rOO}_{-56}, \mathrm{rOO}_{+} 192$ \\
\hline TOTAL & 257 & 87 & 82 & 20 roo insertions \\
\hline
\end{tabular}




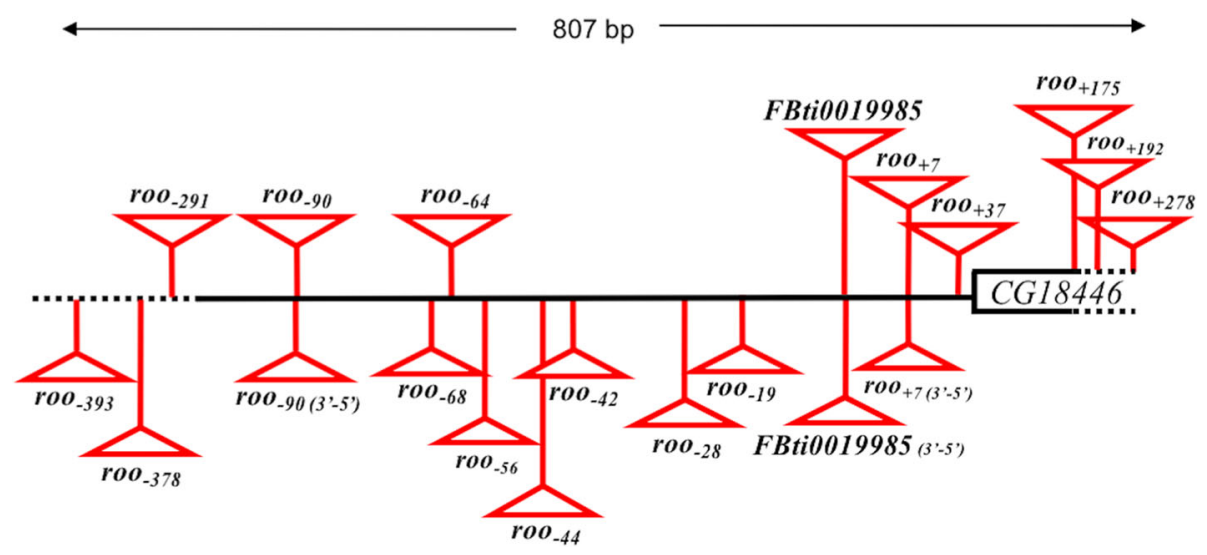

Fig. 1 Twenty roo solo-LTR insertions are located in the promoter region of CG18446 gene in different natural strains. Schematic representation of the CG18446 promoter region where the 20 roo solo-LTRs are inserted. The black line represents the CG18446 promoter region. Note that although only one insertion was found in any given strain, we have represented them together for simplicity. The white box represents CG18446 $5^{\prime} \cup T R$. Regions depicted with dotted lines are not drawn to scale. Insertions present in 5'-3' orientation are shown above the black line and insertions present in $3^{\prime}-5^{\prime}$ orientation are shown below the black line

have been lost in the isofemale strains since they were originally sequenced. Errors in genotyping of T-lex2 could also explain some of these discrepancies, although all the homozygous insertions that T-lex2 predicted were validated by PCR (Additional file $2 \mathrm{~B}$ ).

The majority of the 20 roo insertions inserted in the CG18446 promoter region were present at very low allelic frequencies, ranging from $0.2 \%$ to $16.5 \%$ (Fig. 2, Additional file 2C). The two most common insertions were $\mathrm{roo}_{-} 90$ and FBti0019985, with allelic frequencies of $16.5 \%$ and $6.3 \%$, respectively (Fig. 2, Additional file 2C). While seven of the insertions were private, $\mathrm{roo}_{-} 68$ and roo 90 were present in nine and 13 out of the 15 populations analyzed, respectively (Additional file 2D). We tested whether European populations at different latitudes differed in the diversity of roo insertions or in the total number of strains containing an insertion. Note that we did not considered the strains from Lund (Sweden) as only four strains were analyzed in this population. We found no correlation between latitude and the number of different roo insertions (Pearson $\mathrm{r}^{2}=$ 0.006 , $p$-value $=0.793$ ), or between latitude and the number of strains with an insertion (Pearson $r^{2}=0.063$, $p$-value $=0.388)$. We also analyzed whether any of the insertions were more frequent in cold, temperate, or arid climates (Additional file 1). We found that $\mathrm{roo}_{-} 90$ was more frequent in arid climates $(p$-value $<0.001)$ and roo -64 was more frequent in cold climates $(p$-value $=0.003)$ (Fig. 2).

Overall, we identified 20 roo insertions in the CG18446 promoter region, most of them present at low population frequencies. While the majority of strains (169 out of 257) had one of the 20 roo insertions, none of the strains analyzed contained more than one roo insertion.

\section{Recurrent insertion is the most likely explanation for the presence of 20 insertions in the promoter region of CG18446}

We identified the target site duplication (TSD) for 17 of the 20 roo insertions located in the CG18446 promoter region. These 17 roo solo-LTR insertions have different TSDs suggesting that they are independent insertion events (Additional file 3). 15 of the 17 identified TSD were five bp-long and the consensus TSD was similar to the one previously described [10, 20, 21] (Additional file 3). Thus, multiple insertions in the CG18446 promoter region are likely the result of transposition rather than small rearrangements such as duplications or inversions, which would change the location of the insertions but not the TSDs.

We tested whether the multiple insertions could have been the results of a burst of transposition. We constructed a phylogenetic tree for the roo insertions present in the reference genome, and the 20 roo insertions found in the CG18446 promoter region (see Material and Methods). Briefly, we estimated the unique number of substitutions shared between the two closest TEs assuming that all the roo copies present in the genome derived from a common ancestral sequence [22]. We found four groups of roo copies that are identical to each other and thus appeared to be the result of several bursts of transposition (Fig. 3, see Material and Methods). This is consistent with roo being one of the most active families in the $D$. melanogaster genome $[14,15,23,24]$. 


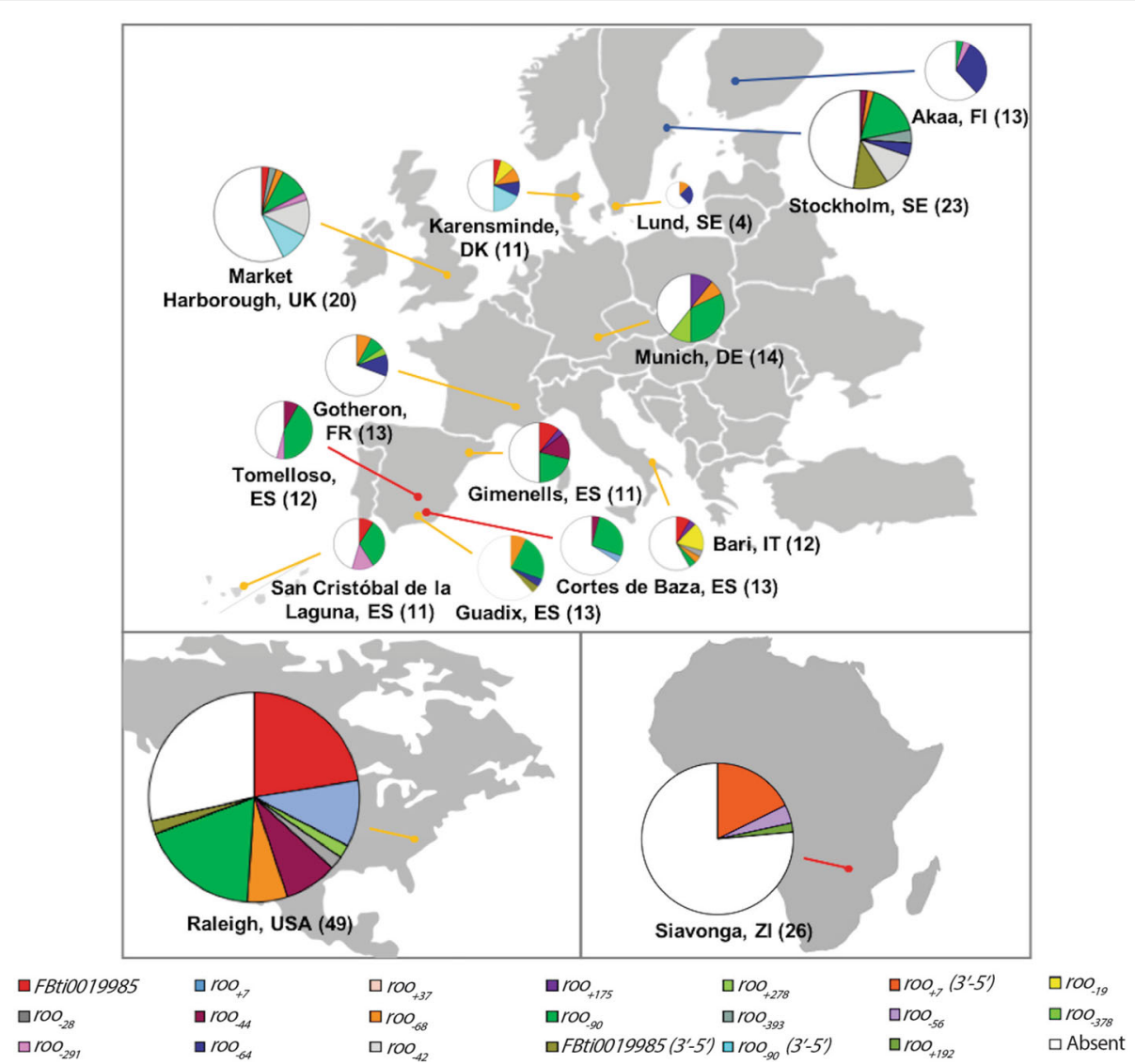

Fig. 2 The majority of the insertions in the CG18446 promoter region are present at very low allelic frequencies. Allelic frequencies of the 20 roo soloLTRs insertions in populations from Europe, North America, and Africa. The number of strains analyzed in each population is given in parenthesis and the pie chart size is proportional to this number. Blue, yellow, and red lines represent populations with cold, temperate, and arid climates, respectively

We then checked whether roo elements have a preference for inserting in $5^{\prime}$ gene regions. We considered as a $5^{\prime}$ gene region the $1 \mathrm{~kb}$ upstream of a gene and its 5'UTR region. Considering not only the 138 roo insertions annotated in the reference genome but also the 12,745 roo de novo insertions found in 177 DGRP strains by TIDAL software [15], we found that only $4.5 \%$ (586) of the roo elements are inserted in gene promoter regions or/and 5'UTR regions (see Material and Methods). This percentage is smaller than the one found for other TE families with preference for inserting in $5^{\prime}$ gene regions, such as the P-element family for which this percentage is $>77 \%[9,10]$. Thus, we considered that roo elements do not have a preference for inserting in $5^{\prime}$ gene regions.

We also checked whether the promoter region of CG18446 has similarities with the promoter of $h s p$ genes that could explain the high number of insertions in this region [8]. We found that, similar to $h s p$ genes, CG18446 is regulated by polymerase pausing [25], and has a high germline transcription activity [13]. Thus, chromatin accessibility could be one of the factors explaining the high TE density in the CG18446 promoter region.

Finally, we found that transcription factor binding sites, core promoter motifs, and Matrix Associated Regions (MARs) previously described in the roo family were highly conserved in all the roo sequences described in this work (Additional file 4) [10, 26, 27].

Overall, we found that the presence of the 20 roo insertions in the CG18446 promoter region is likely to be the result of several bursts of transposition (Fig. 3). Thus, recurrent insertions seem the most likely explanation for the presence of roo elements in the CG18446 promoter region. Similar to the cluster of P-element insertions in the promoter of $h s p$ genes, roo elements are also inserted in a promoter region with an open chromatin architecture [8].

\section{The roo insertion cluster in CG18446 is unique}

We tested whether other roo clusters in gene promoter regions were present in the reference genome. Out of the 137 other roo elements present in the reference genome, 26 are inserted in promoters (less than $1 \mathrm{~kb}$ 


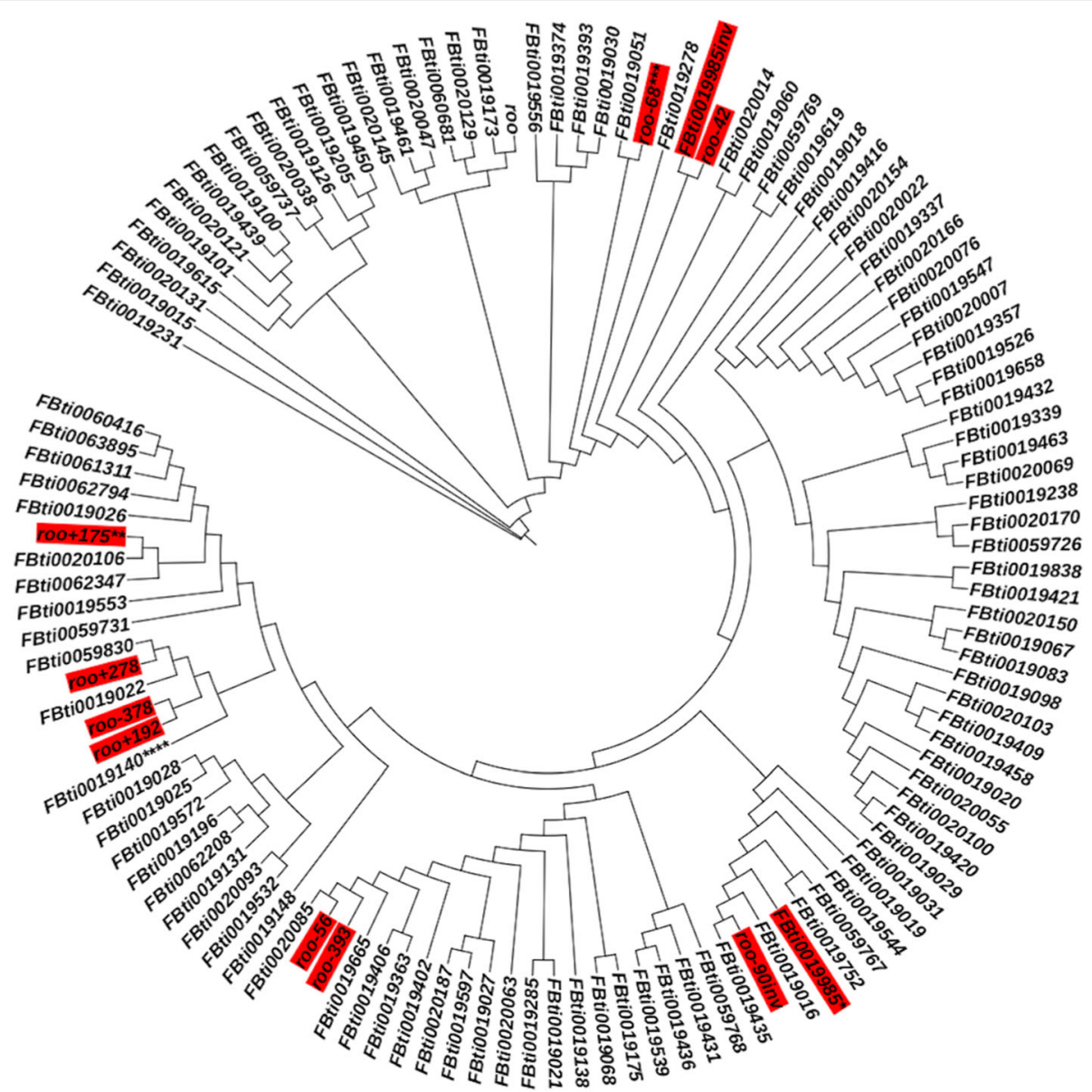

Fig. 3 The 20 roo solo-LTR insertions found in CG18446 promoter region are the result of several bursts of transposition. Phylogenetic tree including the 20 roo solo-LTR insertions found in CG18446 promoter region and 115 other roo insertions annotated in the reference genome. roo solo-LTRs found in this work are highlighted in red. When several TEs with the same exact sequence were identified, we removed all of them but one. The TEs included in the tree are marked with *. The TEs that were eliminated are: $\left(^{*}\right)$ FBti0019017, FBti0019394, FBti0019438, FBti0020009,

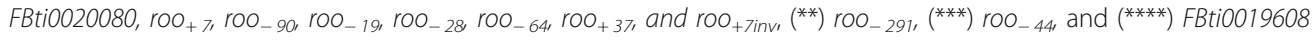

from a gene) or in 5'UTR regions. These 26 roo elements are inserted in 26 different promoter regions, and five of them are solo LTRs (Additional files 5 and 6A). We screened by PCR the presence/absence of insertions in these 26 promoter regions in 10 randomly chosen DGRP strains (see Material and Methods). For 22 of the 26 promoter regions, no other insertion was found in any of the 10 strains. The other four promoter regions contained the same roo element present in the reference genome in all the 10 strains analyzed (Additional files 5 and 6A). These results suggest that considering all the roo insertions annotated in the reference genome only the CG18446 promoter region contain a cluster of roo insertions.

Besides the roo insertions annotated in the reference genome, we also analyzed all the de novo roo insertions identified by TIDAL in a set of 177 DGRP strains $[15,16]$. There are 559 roo elements inserted in promoters or in 5'UTR regions. These 559 roo elements are distributed in
421 gene promoter or $5^{\prime}$ UTR regions (Additional file 5). According to TIDAL, the promoter region of CG18446 has a roo insertion in eight different DGRP strains. We focused on the 27 gene promoter regions where TIDAL identifies three or more strains containing a roo insertion (Additional files 5, 6B and C). In order to test whether any of the 27 promoter regions harbors different roo insertions, we checked by PCR and sequenced the obtained bands of the 27 gene promoters in 95 strains (Additional file $6 \mathrm{~B}$ ). Among the 27 promoter regions analyzed, only four have two different roo insertions in different strains (Table 2). For these four genomic regions, we performed further PCR analysis in another 10 randomly chosen strains. We could not detect any other roo insertion in these promoter regions, suggesting that they probably harbor only the two de novo roo insertions found before.

Finally, it could be that roo insertions tend to form clusters, but that these clusters are deleterious when located in promoter regions. We thus also checked whether roo 
elements cluster in $1 \mathrm{~kb}$ regions genome-wide, not necessarily located in gene promoters. We found five $1 \mathrm{~kb}$ regions with seven or more de novo roo insertions located in chromosomes 2 and 3 (Additional file 7, 8A and B). Because TIDAL does not predict the exact insertion site but rather provides a range of nucleotides where the TE is inserted, it is likely that the total number of roo insertions predicted in these windows is an overestimate. Indeed, the two regions with more roo insertions, 17 and 13 insertions, overlapped $911 \mathrm{bp}$ and $323 \mathrm{bp}$ respectively with the roo cluster in CG18446 promoter region. Based on the screening reported in this work, we know that there are eight and one insertions respectively in these two regions. We checked by PCR whether all the elements predicted within the five $1 \mathrm{~kb}$ regions with more than seven insertions, and two randomly chosen windows with six and four predicted insertions had the same insertion site or not. The two regions overlapping with the CG18446 promoter region contained five and one insertion (Additional file $8 \mathrm{~A}$ ). The other five regions analyzed contained at most two roo insertions (Additional file 8A). Thus, we found that only the $1 \mathrm{~kb}$ region that overlaps with the CG18446 promoter region is actually a roo insertional cluster (Additional file 8A).

\section{Flies with two roo insertions in the CG18446 promoter regions are viable and show similar fecundity rates as flies with one roo insertion}

As mentioned above, none of the 257 strains analyzed contains more than one roo insertion in the CG18446 promoter region. The two roo insertions that are present at higher population frequencies are FBti0019985 and $r o_{-}$90. Thus, for these two insertions, and depending on the population analyzed, we would expect to find from $0.6 \%$ to $8.8 \%$ of flies containing these two insertions in different haplotypes (Additional file 2E). Since the number of strains sampled per population is not very high (Additional file 1), it could be that we have not screened enough flies to find one strain containing two insertions.

To discard that flies with two roo insertions have reduced egg-to-adult viability or reduced fecundity compared with flies containing only one roo insertion, we created flies containing two insertions in the CG18446 promoter region (see Material and Methods). We found that flies with two roo insertions had similar or significantly higher viability compared with flies with only one of the roo insertions (ANOVA p-value $<0.001$ Fig. 4a). Early fecundity of flies containing two roo insertions was not significantly different from that of flies containing only one roo insertion (ANOVA $p$-value $=0.068$, Fig. 4b). Similarly, we did not find differences in the average number of eggs laid per day during 18 days between flies with one or two roo insertions (ANOVA $p$-value $=0.494$, Fig. 4c). Note that the genetic background of flies containing one or two roo insertions is different. Thus, polymorphisms other than the presence/absence of these insertions are likely to be also contributing to the lack of differences observed.

\section{Discussion}

Besides the nine roo solo-LTRs found in Merenciano et al. (2016) [10], we have discovered 11 new roo insertions in the CG18446 promoter region. It is known that $D$. melanogaster populations differ in their $\mathrm{TE}$ content [10, 28-31]. Thus, it could be that analyzing more populations, especially from geographical areas currently underrepresented such as Central and South America or Asia, could lead to the discovery of more roo insertions in the CG18446 promoter region. However, the number of populations analyzed in this work was seven-fold higher than in Merenciano et al. (2016) [10] and the number of new roo insertions was only twice that of our previous study, suggesting that it is likely that we have discovered the majority of the roo elements in the CG18446 promoter region.

All 20 roo insertions identified in the promoter region of CG18446 are solo LTR insertions, while the majority (21 out of 26) of the roo insertions found in other promoter regions are full-length insertions (Additional file 6 A). Solo LTRs are presumably the result of homologous unequal recombination between the two LTRs of a full-length element [14]. Thus, the recombination region where these TEs are located could influence whether they are full-length elements or solo LTRs. However, only four of the 21 full-length elements are present in regions with a low recombination rate, while the other 17 roo insertions are located in regions with a similar recombination rate as the CG18446 promoter region (Additional file 6A) [32, 33]. Although it is not clear why all the insertions in the CG18446 promoter region are solo LTRs, the location of this promoter in an open chromatin region could be one of the

Table 2 de novo roo insertions found in four gene promoter regions

\begin{tabular}{lll}
\hline Promoter region & Number of strains predicted to have an insertion & Genomic coordinates of the insertion sites validated \\
\hline plum & 4 & $3 R: 25,621,076$ and $26,521,553$ \\
CG11459 & 3 & $3 R: 6,027,532$ and $6,027,608$ \\
CG15879 & 3 & $3 L: 2,169,152$ and $2,169,162$ \\
CR44657 & 3 & $X: 14,114,700$ and $14,115,661$ \\
\hline
\end{tabular}




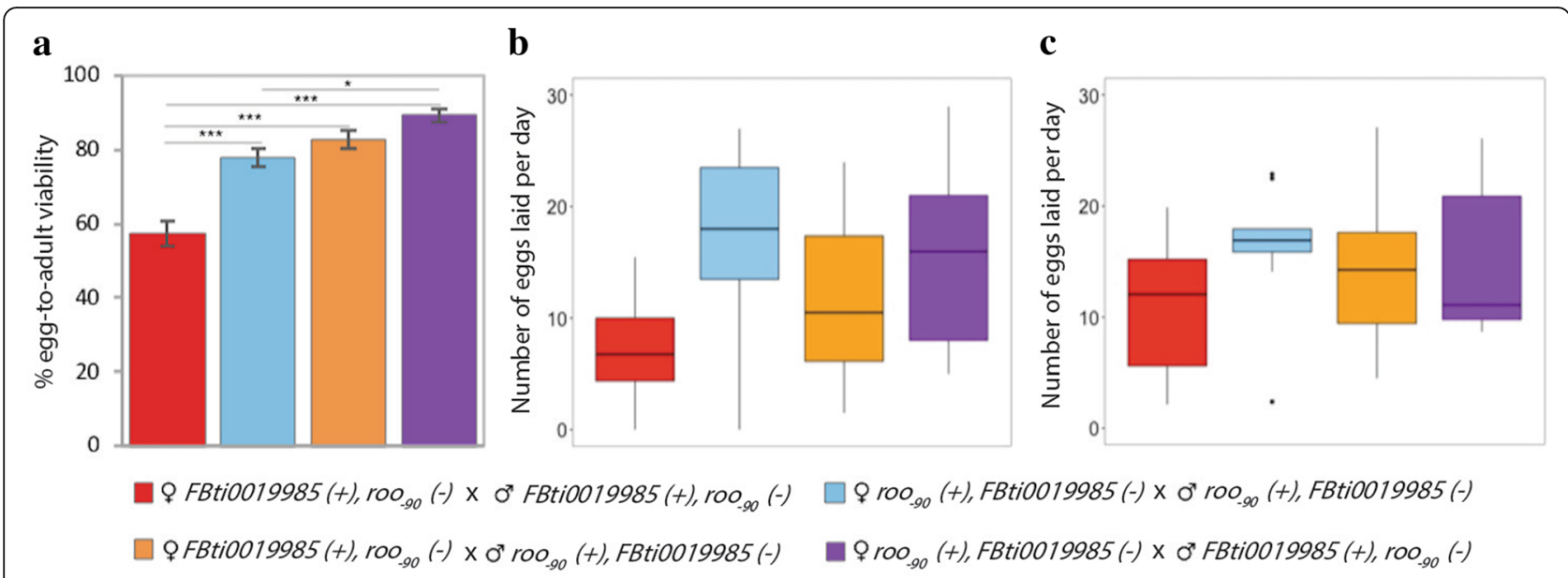

Fig. 4 Egg-to-adult viability and fecundity are not likely to reduce the probability of finding flies with more than one roo element in the CG18446 promoter region. a. Percentage of emerged flies from the four crosses tested. $\mathbf{b}$. Average number of eggs laid per day during the first $48 \mathrm{~h}$ for each of the four crosses analyzed. c. Average number of eggs laid per day during 18 days for each of the four crosses analyzed

contributing factors as it has been suggested that chromatin accessibility favors double strand breaks and thus recombination [33].

Phylogenetic analyses revealed that the presence of multiple roo insertions in the CG18446 promoter is likely to be the result of several bursts of transposition rather than small rearrangements or insertion preference for $5^{\prime}$ gene flanking regions. This is consistent with previous data suggesting that roo is one of the most active TE families with a high transposition rate $[14,15,23,24]$. Indeed, it has been suggested that roo elements have been able to evade piRNA silencing, because the number of novel roo insertions is high despite the presence of a high proportion of piRNAs against this family [15]. Note that the piRNA data analyzed in Rahman et al. (2015) [15] was obtained from ovaries and ovarian cell cultures [34, 35], and it has been suggested that TE activity in female and male germlines might differ due to polymorphisms in the piRNA regulatory genes between sexes [36].

Why roo insertions recurrently insert in the promoter region of the CG18446 gene is not yet completely understood. We showed that there is no other cluster of roo insertions in promoter regions or in 1 $\mathrm{kb}$ genomic regions genome-wide. Thus, the presence of multiple roo insertion in this particular promoter region is probably related to some specific feature of this promoter. We indeed found that chromatin accessibility could be one of the factors explaining the recurrent insertions in this promoter region. In $D$. melanogaster, one other insertional cluster described is also located in the promoter region of stress response genes, which is located in an open chromatin region [8]. Several of the TEs located in the promoter of $h s p$ genes have been shown to affect the expression of the nearby genes by altering the promoter architecture [7, 37]. So far, only one of the roo insertions in the CG18446 promoter region, FBti0019985, has been shown to affect the expression of this gene by adding a new transcription start site [10]. In this work, we found that $\mathrm{roo}_{-} 90$ has an allelic frequency of $16.5 \%$ and is significantly more frequent in arid climates. Thus, it would be interesting to test whether this insertion affects expression of the nearby gene and/or is associated with a fitness-related trait that could explain its higher frequencies in arid climate conditions.

Finally, in Arabidopsis thaliana recurrent insertion of TEs from the Copia family in the first intron of the FLC locus have been associated with epigenetic regulation of this locus in response to cold [38]. Thus, not only in D. melanogaster but at least also in A. thaliana, recurrent insertions of TEs belonging to a single family are associated with stress-related genes, and some of these insertions have fitness-related consequences.

\section{Material and methods \\ Fly stocks}

Fly stocks used for PCR screening are listed in Additional file 2A. One outbred population homozygous for the presence of FBti0019985, and one outbred population homozygous for the presence of $\mathrm{roo}_{-} 90$ were generated by a round-robin cross of inbred lines from the Drosophila Genetic Reference Panel (DGRP) [39] and isofemale lines from different European populations (Additional file 9). We maintained the population by random mating with a large population size for over five generations before starting the experiments. All flies were reared on fly food medium in a 12:12 h light/dark cycle at $25^{\circ} \mathrm{C}$. 


\section{Analysis of TE presence/ absence}

We used the same PCR approach as in Merenciano et al. (2016) [10] to check for the presence/absence of TE insertions in the CG18446 promoter region in 234 natural strains from Europe, North America (DGRP) [16] and Africa (Nexus) [17]. Briefly, genomic DNA was extracted from a pool of 10 female flies of each strain. We performed PCR with two primer pairs. Primer pair Flanking (FL6) (5'-AACAATGCAAGTCCGTGCTC-3') and Right (R) (5'-CGTAGGATCAGTGGGTGAA AATG-3') are expected to give an 802 bp band when insertions are absent and a bigger band when there is an insertion. Primer pair Left (L) (5' -AGTCCCTTA GTGGGAGACCACAG-3') and R are expected to give a band only when there is a roo insertion. When the two PCRs failed, we used the alternative primer R2 (5' -CGGGTACATCTTTGCGGGAT-3'). When the PCR using the FL6 primer failed, we used the alternative primers FL (5'-GGCATCATAAAACCGTTGA ACAC-3'), and/or FL7 (5' - TTCGTGCGTGTTCGGT ACTT-3'). PCR products were purified using the NucleoSpin ${ }^{\circ}$ Gel and PCR Clean-up kit (Macherey-Nagel) using the manufacturer's instructions and Sanger-sequenced using FL and/or L and R primers to verify the results. PCR failed for 16 strains and thus we could analyze 218 out of the 234 .

\section{Consensus motifs}

We aligned using Genious 9.1.4 (https://www.geneious. com) the roo element sequences from the 114 strains that were fully sequenced in this work. We also included in the alignments the roo sequences reported in Merenciano et al. (2016) [10]. We identified in these sequences the nine transcription factor binding sites, the Inr promoter motif, and the MARs previously identified by Merenciano et al. (2016) [10]. We constructed the consensus sequence logos using WebLogo [40]. The target site duplication (TSD) consensus was constructed also using Weblogo with 15 out of 17 of the TSDs found in this work and in Merenciano et al. (2016) [10]. The two TSD removed have shorter sequence length.

\section{Phylogenetic analysis}

We followed the same approach as in Merenciano et al. (2016) [10]. Briefly, 16 of the 20 roo solo-LTR insertions in CG18446 promoter region were sequenced in several strains (Additional file 2A). For each of these 16 insertions, we aligned the sequences and generated a consensus. We then aligned these 16 consensus sequences, the other four roo insertions and the 115 roo insertions found in $D$. melanogaster genome using the multiple sequence aligner program MAFFT. We inferred a maximum likelihood tree under the general time-reversible nucleotide model and a gamma distribution of evolutionary rates, using RAxML Version 8 [41] (Additional file 10). We removed from the phylogenetic analysis those TEs with exact identical sequences. The interactive tree of life (iTOL) framework (https://ito l.embl.de/) was used for the analysis and visualization of the tree, ignoring branch lengths.

\section{Analysis of other roo clusters in promoter regions}

We analyzed the region where 27 roo elements are inserted less than $1 \mathrm{~kb}$ from a gene or in $5^{\prime} \mathrm{UTR}$ regions in the D. melanogaster reference genome (R6.07) in 10 randomly chosen DGRP strains. To determine if 10 strains are enough to detect a cluster, we ran 1000 randomly generated trials using a Python script. This script randomly chose 10 strains among all the DGRP strains screened by PCR in this work and in Merenciano et al. (2016) [10] and counted the number of different roo insertions obtained in every iteration. We found that four was the average number of different roo insertions that can be found in a screening of 10 randomly chosen DGRP strains. Then, by checking 10 different DGRP strains we expected to find an average of four different roo insertions in the case of the presence of an insertion cluster similar to the one found in the CG18446 promoter region.

For each strain, genomic DNA was extracted from a pool of 10 female flies. Primers (forward and reverse) were design in the flanking region of the insertion amplifying a minimum of $500 \mathrm{bp}$ when the TE is not present (Additional file 11). We also used a combination of primers (roo_primer and reverse) that gave a PCR band only when a roo element is present (Additional file 11). PCR programs were set according to the length of each TE insertion. In addition, we also considered de novo insertions found with TIDAL software in a set of 177 DGRP strains [15]. We first selected all the 559 roo elements predicted to be inserted less than $1 \mathrm{~kb}$ from the nearest gene or in 5'UTR regions. Then, we grouped the insertions based on the promoter region where they are inserted. Finally, we analyzed by PCR the 27 promoter regions where three or more strains putatively have a roo insertion. As before, genomic DNA was extracted from a pool of 10 female flies of each strain. Five combinations of primer pairs were used in order to verify the position of the insertion: one primer pair in the flanking region of the insertion amplifying a minimum of $500 \mathrm{bp}$ when the TE is not present (ClusterF and ClusterR), and other four combinations where one primer was located in the LTR region in both genomic orientations (ClusterF and rooL2, ClusterR and rooL, ClusterF and rooL, and ClusterR and rooL2) (Additional file 11). PCR products were purified and Sanger sequenced as mentioned before. For the four promoter regions for which we found two roo insertions, $8,13,17$, and 23 , we 
performed additional PCRs following the same approach in ten DGRP strains (RAL-105, RAL-129, RAL-136, RAL-161, RAL-208, RAL-239, RAL-208, RAL-239, RAL-280, RAL-301, RAL-309, and RAL-379).

\section{Analysis of other clusters in the genome}

We selected the 12,745 roo de novo insertions predicted by TIDAL software in 177 DGRP strains [15]. Since TIDAL software predicts a range of coordinates where the TEs may be inserted, we established as the insertion site the midpoint of the coordinates. For each chromosome arm (except the Y chromosome), we first considered as the same insertion those inserted within $5 \mathrm{bp}$ windows. Thus, we got a total of 9243 roo de novo insertions. After that, we counted how many predicted de novo roo elements are in windows of $1 \mathrm{~kb}$. We then chose for PCR validation five $1 \mathrm{~kb}$ regions with more than seven predicted roo insertions, and two additional $1 \mathrm{~kb}$ regions with four and six insertions predicted. Every region was validated in 7-10 different DGRP strains. For each strain, genomic DNA was extracted from a pool of 10 female flies. Five combinations of primers were designed following the same approach as before (Additional file 11). PCR products were purified and Sanger sequenced as mentioned before.

\section{Expected genotype frequency calculation}

For all the populations analyzed in this work, the expected genotype frequencies of flies containing both FBti0019985 and $\mathrm{roo}_{-90}$ insertions were calculated multiplying the observed allelic frequency for FBti0019985 and the observed allelic frequency for roo 90, considering that they are in different haplotypes (Additional file 2E).

\section{Viability assays}

We checked the egg-to-adult viability of outbred FBti0019985 (+) crosses, outbred roo ${ }_{-90}(+)$ crosses and their reciprocal crosses. In total, 100 five to seven day-old flies (50 males and 50 virgin females) for each cross were allowed to lay eggs for $24 \mathrm{~h}$ on apple juice-agar medium with fresh yeast at $25^{\circ} \mathrm{C}$. Embryos were collected following the protocol described in Schou et al. (2013) [42]. For each cross, we collected a total number of 150 embryos and put them in groups of 30 in empty vials with fresh food. We maintained the vials at $25^{\circ} \mathrm{C}$ until adult emergence. The percentage of egg-to-adult viability was calculated as the ratio of the number of emerged flies to the total number of embryos placed in each vial. Statistical significance was calculated performing ANOVA using SPSS v. 21 followed by Tukey post-hoc multiple comparison procedure.

\section{Fecundity assays}

We checked the fecundity of outbred FBti0019985 (+) crosses, outbred roo $_{-90}(+)$ crosses and their reciprocal crosses. For each cross, 10 virgin females were placed individually with one male in vials with fresh food. Flies were moved to new vials every day during 18 days without $\mathrm{CO}_{2}$ anesthesia, and dead males were replaced. The number of eggs laid per day was counted every day during this period. The average of the total number of eggs laid per day during the 18 days (total fecundity), and the average of the total number of eggs laid per day during the first $48 \mathrm{~h}$ (early fecundity) was compared between crosses. We removed from the analysis those vials where the female died during the experiment. Statistical significance was calculated performing ANOVA using SPSS v.21.

\section{Additional files}

Additional file 1: Populations used for the analysis. (XLSX $11 \mathrm{~kb}$ )

Additional file 2: A. PCR results for the 277 strains analyzed in this work and in Merenciano et al. (2016). Strains used in Merenciano et al. (2016) are highlighted in blue. B. Tlex-2 predictions in Merenciano et al. (2016) compared to PCR results in this work. Correct predictions are highlighted in green. Strains with roo insertions not identified in Merenciano et al. (2016) are highlighted in orange. Strains for which no results were obtained either by T-lex2 or by PCR are highlighted in grey. C. Allelic frequencies of all the 20 roo insertions in all the populations analyzed. EU: Europe, NA: North America and ZI: Zambia. D. Allelic frequencies (\%) of the 20 roo insertions in the 15 different populations analyzed. Elements only present in one population are highlighted in red. $\mathbf{E}$. Expected genotype frequency of heterozygous flies with the two most common insertions, FBti0019985 and roo-90 in all the populations analyzed. a: FBti0019985 alellic frequency, b: roo-90 alellic frequency, and c: absent alellic frequency. (XLSX $47 \mathrm{~kb}$ )

Additional file 3: A. Consensus target site duplication (TSD) sequence identified in Merenciano et al. (2016) (left panel) and consensus TSD identified with the data of this paper and Merenciano et al. (2016) (right panel). B. TSD sequences of the 20 roo insertions. Frequency represents the number of strains that harbor the TSD out of the number of strains with a complete sequenced region. (DOCX $332 \mathrm{~kb}$ )

Additional file 4: Consensus sequence of the transcription factor binding sites and matrix attachment regions identified in all the roo sequences identified in the CG18446 promoter region. (DOCX $330 \mathrm{~kb}$ )

Additional file 5: The formation of roo insertional clusters in gene promoter regions is not a roo family characteristic. Scheme of the gene promoter regions containing roo elements present in the reference genome (left) and present in 177 DGRP inbred strains (right). (DOCX 29 kb)

Additional file 6: A. Coordinates (R6), length, recombination rates and $P C R$ results of the 26 promoter regions with a roo insertion in the reference genome. B. PCR results and de novo TE information of the 28 promoter regions where $>=3$ strains putatively have a roo insertion based on TIDAL software predictions. C. Promoter regions where $<3$ strains putatively have a roo insertion based on TIDAL software predictions. (XLSX $61 \mathrm{~kb}$ )

Additional file 7: Genome-wide distribution of de novo roo elements found in 177 DGRP strains. Number of predicted de novo roo elements found in 177 DGRP strains inserted in $1 \mathrm{~kb}$ windows in chromosomes 2, 3, 4, and X. (DOCX $102 \mathrm{~kb})$

Additional file 8: A. PCR results of the five $1 \mathrm{~kb}$ regions with more roo insertions predicted by TIDAL software. B. $1 \mathrm{~kb}$ regions with at least 1 roo insertion predicted by TIDAL software. Regions checked by PCR are highlighted in yellow. (XLSX 166 kb) 
Additional file 9: Schematic representation of the round-robin crossdesign for outbred FBti0019985 (+), FBti0019985 (-), roo 90 (+), and roo 90 (-) generation. (DOCX $123 \mathrm{~kb}$ )

Additional file 10: Phylogenetic tree of the 20 roo solo-LTR found in CG18446 promoter region and 115 other roo insertions annotated in the reference genome. (TXT $6 \mathrm{~kb}$ )

Additional file 11: List of primers used for insertional cluster validation. (XLSX $12 \mathrm{~kb})$

\section{Abbreviations}

DGRP: Drosophila Genetic Reference Panel; LTR: Long Terminal Repeat; TE: Transposable Element; TSD: Target Site Duplication

\section{Acknowledgements}

We thank members of the González lab for providing comments on the manuscript. We also thank members of the DrosEU consortium (droseu.net) for sharing isofemale lines from their lab collections.

\section{Funding}

This work was funded by the European Commission (H2020-ERC-2014-CoG647900). C.I. was funded by an ERASMUS+ fellowship. We acknowledge the support of the Secretaria d'Universitats i Recerca del Departament d'Economia i Coneixement de laGeneralitat de Catalunya (GRC 2017 SGR 880). We also acknowledge support of the publication fee by the CSIC Open Access Publication Support Initiative through its Unit of Information Resources for Research (URICI).

The funding body had no role in the design of the study and collection, analysis, and interpretation of data and in writing the manuscript should be declared.

\section{Availability of data and materials}

The datasets generated as part of the study are available as supplementary information.

\section{Authors' contributions}

MM designed the study, acquired, analyzed and interpreted the data, and wrote the manuscript. Cl acquired and analyzed the data. JG designed the study, interpreted the data, and wrote the manuscript. All the authors approved the final manuscript.

Ethics approval and consent to participate

Not applicable.

\section{Consent for publication}

Not applicable.

\section{Competing interests}

The authors declare that they have no competing interests.

\section{Publisher's Note}

Springer Nature remains neutral with regard to jurisdictional claims in published maps and institutional affiliations.

\section{Author details}

${ }^{1}$ Institute of Evolutionary Biology (CSIC-Universitat Pompeu Fabra), Passeig Maritim de la Barceloneta 37,49, 08003 Barcelona, Spain. ²Department of Life Sciences and Systems Biology, University of Turin, Turin, Italy.

Received: 21 December 2018 Accepted: 28 February 2019 Published online: 13 March 2019

\section{References}

1. Bergman CM, Quesneville $H$, Anxolabehere D, Ashburner M. Recurrent insertion and duplication generate networks of transposable element sequences in the Drosophila melanogaster genome. Genome Biol. 2006; 7(11):R112.

2. Makałowski W, Pande A, Gotea V, Makałowska I. Transposable elements and their identification. Methods Mol Biol. 2012;855:337-59.
3. Ewing AD. Transposable element detection from whole genome sequence data. Mob DNA. 2015:6:24

4. Modolo L, Lerat E. In: Poptsova MS, editor. Identification and Analysis of transposable elements in genomic sequences. U.K: Caister Academic Press; 2014

5. Cridland JM, Macdonald SJ, Long AD, Thornton KR. Abundance and distribution of transposable elements in two Drosophila QTL mapping resources. Mol Biol Evol. 2013;30(10):2311-27.

6. King EG, Macdonald SJ, Long AD. Properties and power of the Drosophila synthetic population resource for the routine dissection of complex traits. Genetics. 2012;191(3):935-49.

7. Lerman DN, Michalak P, Helin AB, Bettencourt BR, Feder ME. Modification of heat-shock gene expression in Drosophila melanogaster populations via transposable elements. Mol Biol Evol. 2003;20(1):135-44.

8. Walser JC, Chen B, Feder ME. Heat-shock promoters: targets for evolution by P transposable elements in Drosophila. PLoS Genet. 2006:2(10):e165.

9. Spradling AC, Stern DM, Kiss I, Roote J, Laverty T, Rubin GM. Gene disruptions using $\mathrm{P}$ transposable elements: an integral component of the Drosophila genome project. Proc Natl Acad Sci U S A. 1995;92(24):10824-30.

10. Merenciano M, Ullastres A, de Cara MA, Barron MG, Gonzalez J. Multiple independent Retroelement insertions in the promoter of a stress response gene have variable molecular and functional effects in Drosophila. PLoS Genet. 2016;12(8):e1006249.

11. Telonis-Scott M, Hallas R, McKechnie SW, Wee CW, Hoffmann AA. Selection for cold resistance alters gene transcript levels in Drosophila melanogaster. J Insect Physiol. 2009;55(6):549-55.

12. Kong EC, Allouche L, Chapot PA, Vranizan K, Moore MS, Heberlein U, et al. Ethanol-regulated genes that contribute to ethanol sensitivity and rapid tolerance in Drosophila. Alcohol Clin Exp Res. 2010;34(2):302-16.

13. Gramates LS, Marygold SJ, Santos GD, Urbano JM, Antonazzo G, Matthews BB, et al. FlyBase at 25: looking to the future. Nucleic Acids Res. 2017:45(D1): D663-D71.

14. Kaminker JS, Bergman CM, Kronmiller B, Carlson J, Svirskas R, Patel S, et al. The transposable elements of the Drosophila melanogaster euchromatin: a genomics perspective. Genome Biol. 2002;3(12):RESEARCH0084.

15. Rahman R, Chirn GW, Kanodia A, Sytnikova YA, Brembs B, Bergman CM, et al. Unique transposon landscapes are pervasive across Drosophila melanogaster genomes. Nucleic Acids Res. 2015;43(22):10655-72.

16. Huang W, Massouras A, Inoue Y, Peiffer J, Ramia M, Tarone AM, et al. Natural variation in genome architecture among 205 Drosophila melanogaster genetic reference panel lines. Genome Res. 2014;24(7):1193-208.

17. Lack JB, Cardeno CM, Crepeau MW, Taylor W, Corbett-Detig RB, Stevens KA, et al. The Drosophila genome nexus: a population genomic resource of 623 Drosophila melanogaster genomes, including 197 from a single ancestral range population. Genetics. 2015;199(4):1229-41.

18. Roiha H, Rubin GM, O'Hare K. P element insertions and rearrangements at the singed locus of Drosophila melanogaster. Genetics. 1988;119(1):75-83.

19. Shilova $\mathrm{VY}$, Garbuz DG, Myasyankina EN, Chen B, Evgen'ev MB, Feder ME, et al. Remarkable site specificity of local transposition into the Hsp70 promoter of Drosophila melanogaster. Genetics. 2006;173(2):809-20.

20. Fiston-Lavier AS, Barron MG, Petrov DA, Gonzalez J. T-lex2: genotyping, frequency estimation and re-annotation of transposable elements using single or pooled next-generation sequencing data. Nucleic Acids Res. 2015;43(4):e22.

21. Linheiro RS, Bergman CM. Whole genome resequencing reveals natural target site preferences of transposable elements in Drosophila melanogaster. PLoS One. 2012;7(2):e30008.

22. Rech GE, Bogaerts-Márquez M, Barrón MG, Merenciano M, Villanueva-Cañas J, Horváth V, et al. Stress response, behavior, and development are shaped by transposable element-induced mutations in Drosophila. PLoS Genet 2019:15(2):e1007900

23. Papaceit M, Avila V, Aguade M, Garcia-Dorado A. The dynamics of the roo transposable element in mutation-accumulation lines and segregating populations of Drosophila melanogaster. Genetics. 2007;177(1):511-22.

24. Diaz-Gonzalez J, Vazquez JF, Albornoz J, Dominguez A. Long-term evolution of the roo transposable element copy number in mutation accumulation lines of Drosophila melanogaster. Genet Res (Camb). 2011;93(3):181-7.

25. Saunders A, Core $\amalg$, Sutcliffe C, Lis JT, Ashe HL. Extensive polymerase pausing during Drosophila axis patterning enables high-level and pliable transcription. Genes Dev. 2013;27(10):1146-58.

26. Juven-Gershon T, Kadonaga JT. Regulation of gene expression via the core promoter and the basal transcriptional machinery. Dev Biol. 2010:339(2): 225-9. 
27. Mamillapalli A, Pathak RU, Garapati HS, Mishra RK. Transposable element 'roo' attaches to nuclear matrix of the Drosophila melanogaster. J Insect Sci. 2013;13:111.

28. Gonzalez J, Lenkov K, Lipatov M, Macpherson JM, Petrov DA. High rate of recent transposable element-induced adaptation in Drosophila melanogaster. PLoS Biol. 2008;6(10):e251.

29. Hill T, Schlotterer C, Betancourt AJ. Hybrid dysgenesis in Drosophila simulans associated with a rapid invasion of the P-element. PLoS Genet. 2016;12(3):e1005920.

30. Bergman CM, Han S, Nelson MG, Bondarenko V, Kozeretska I. Genomic analysis of $\mathrm{P}$ elements in natural populations of Drosophila melanogaster. PeerJ. 2017:5:e3824.

31. Lerat E, Goubert C, Guirao-Rico S, Merenciano M, Dufour AB, Vieira C, et al. Population specific dynamics and selection patterns of transposable element insertions in European natural populations. Mol Ecol. 2018.

32. Fiston-Lavier AS, Carrigan M, Petrov DA, González J. T-lex: a program for fast and accurate assessment of transposable element presence using nextgeneration sequencing data. Nucleic Acids Res. 2011;39(6):e36.

33. Comeron JM, Ratnappan R, Bailin S. The many landscapes of recombination in Drosophila melanogaster. PLoS Genet. 2012;8(10):e1002905.

34. Song J, Liu J, Schnakenberg SL, Ha H, Xing J, Chen KC. Variation in piRNA and transposable element content in strains of Drosophila melanogaster. Genome Biol Evol. 2014;6(10):2786-98

35. Sytnikova YA, Rahman R, Chirn GW, Clark JP, Lau NC. Transposable element dynamics and PIWI regulation impacts IncRNA and gene expression diversity in Drosophila ovarian cell cultures. Genome Res. 2014;24(12):1977-90.

36. Saint-Leandre B, Clavereau I, Hua-Van A, Capy P. Transcriptional polymorphism of piRNA regulatory genes underlies the mariner activity in Drosophila simulans testes. Mol Ecol. 2017;26(14):3715-31.

37. Lerman DN, Feder ME. Naturally occurring transposable elements disrupt hsp70 promoter function in Drosophila melanogaster. Mol Biol Evol. 2005; 22(3):776-83.

38. Quadrana L, Bortolini Silveira A, Mayhew GF, LeBlanc C, Martienssen RA Jeddeloh JA, et al. The Arabidopsis thaliana mobilome and its impact at the species level. Elife. 2016;5:e15716.

39. Mackay TF, Heinsohn SL, Lyman RF, Moehring AJ, Morgan TJ, Rollmann SM. Genetics and genomics of Drosophila mating behavior. Proc Natl Acad Sci U S A. 2005;102(Suppl 1):6622-9.

40. Crooks GE, Hon G, Chandonia JM, Brenner SE. WebLogo: a sequence logo generator. Genome Res. 2004;14(6):1188-90.

41. Stamatakis A. RAxML version 8: a tool for phylogenetic analysis and postanalysis of large phylogenies. Bioinformatics. 2014;30(9):1312-3.

42. Schou MF. Fast egg collection method greatly improves randomness of egg sampling in Drosophila melanogaster. Fly (Austin). 2013;7(1):44-6.

Ready to submit your research? Choose BMC and benefit from:

- fast, convenient online submission

- thorough peer review by experienced researchers in your field

- rapid publication on acceptance

- support for research data, including large and complex data types

- gold Open Access which fosters wider collaboration and increased citations

- maximum visibility for your research: over $100 \mathrm{M}$ website views per year

At $\mathrm{BMC}$, research is always in progress.

Learn more biomedcentral.com/submissions 